\title{
On the relation between GARCH and stable processes
}

\author{
Casper G. de Vries* \\ Katholieke Universiteit Leuven, B-3000 Leuven, Belgium
}

Received June 1988, final version received March 1990

Stable and GARCH processes have been advocated for modeling financial data. The aim of this note is to compare the two processes. It is shown that the unconditional distribution of variates from a GARCH-like process, which explicitly models the clustering of volatility and exhibits the fat-tail property as well, can be stable. Given suitable conditions the conditional distributions are stable as well. While it is generally realized that processes with variates that have unconditional nonnormal stable densities have a high frequency of 'outliers', it is less well known that they can exhibit the clustering phenomenon too. The clustering is obtained through stable subordination with conditional scaling.

\section{Introduction}

The literature on modeling returns on speculative assets consists of two main approaches. One approach only models the unconditional distribution of the returns, while the other approach also takes the conditional distributional aspects into account. The former approach at first hypothesized a Brownian motion, but this proved untenable due to the slowly declining probability mass in the tails of the empirical distribution function (d.f.) of the innovations. In order to account for this phenomenon, Mandelbrot (1963) in a seminal paper proposed to use the other members of the stable class rather than the normal d.f. While there are other fat-tailed d.f.'s, the stable d.f.'s are the only d.f.'s which are type-invariant under addition (i.e., only the

\footnotetext{
*I am grateful to Ronny Claeys, Feike Drost, Geert Gielens, Laurens de Haan, Rauuf Jä̈bi, Teun Kloek, Paul Kofman, Luc Lauwers, Theo Nijman, Peter Schotman, Guoqiang Tian, and the comments of four anonymous referees. This work was supported by the Texas A\&M University, the Erasmus Universiteit Rotterdam, and the CentER for Economic Research. I benefited from presentations at the E.S.E.M. 1989 in Munich and Hermann Garber's graduate seminar at the University of Zürich.
} 
location and scale may change, while the characteristic exponent is constant), which is a desirable property given that returns are time-additive. ${ }^{1}$ The stable model has become a popular model in several areas of economics [see, e.g., Westerfield (1977) and Akgiray and Booth (1988)]. Nevertheless, other fattailed d.f.'s, like the Student- $t$ [see, e.g., Blattberg and Gonedes (1974)], have been studied because a finite variance is sometimes found to be a characteristic of the data as well [i.e., due to the applicability of the central limit law; see Diebold (1988)]. More recently, discrete mixtures of the normal d.f. and mixed diffusion jump processes are becoming popular [see, e.g., Kon (1984) and Tucker and Pond (1988)], as these processes exhibit the also observed higher-than-normal kurtosis. But note that these models do not have the fat-tail property due to the exponentially declining tails of their density functions.

The other and more recent strand of literature not only considers the unconditional d.f., but also focuses on the conditional distributional aspects. Mandelbrot (1963) already discussed the fact that there are clusters of high and low volatility in the return data. Typically, dependence in the second moment of the returns' d.f. is much stronger than dependence in the first moment. But not until the ARCH model [introduced by Engle (1982)] and the GARCH extension [see Bollerslev and Engle (1986)] have economists come to grips with this stylized fact. In addition to exhibiting the clustering phenomenon, the unconditional d.f.'s of the variates from an ARCH process have fat tails [see, e.g., De Haan et al. (1989)], though the variance is still finite. Understandably the ARCH-type processes have gained wide popularity [cf. Diebold (1988)]. For example, Diebold (1987, p. 3; 1988, ch. 4) and Bollerslev (1987, p. 542) argue in favor of the GARCH process vis-à-vis a process with variates that are unconditionally stable distributed, because the latter process ostensibly lacks the clustering phenomenon.

This begs the question whether it is not possible for a stable proce.; to exhibit the clustering phenomenon. In the existing literature we could not locate a reference dealing with this issue. ${ }^{2}$ The aim of this note is to partly fill this gap by comparing the wo processes. In particular, we jitend to show that there exists a class of GARCH-like processes of which the realizations are unconditionally stable distributed. We also provide an example of a stable process which exhibits clusters of volatility; i.e., all conditional distributions of this process follow a stable law as well. Some hints towards empirical implementation are provided. It follows that, under certain conditions on the parameters of a GARCH-like process, the stable and GARCH processes are

\footnotetext{
${ }^{1}$ Furthermore, due to triangular arbitrage between foreign exchange rates, foreign exchange rate returns are additive as well across different rates.

${ }^{2}$ The only somewhat related paper is by McCulloch (1985), who introduces a process with stable innovations but conditional scaling. The resulting unconditional distributions, however, are not stable. Wolff (1988) compares the ARCH model with the random coefficient model.
} 
observationally equivalent from the viewpoint of the unconditional distribution, and in some cases in all respects. In summary, the various strands of the literature have tried to cope with following stylized facts of returns on financial assets: returns (i) have d.f.'s with fat tails and a higher-than-normal kurtosis, (ii) exhibit clusters of high and low volatility, (iii) are additive such that their distributions are type-invariant, and (iv) normed sums tend to follow a limit law. The unconditional stable literature captures facts (i), (iii), and (iv). The ARCH literature deals with facts (i), (ii), and (iv). This note shows that there are processes which exhibit all four stylized facts, or different subsets of these facts.

\section{The volatility function}

Consider the following slightly modified $\operatorname{GARCH}(1,1)$ process:

$$
\begin{aligned}
& Y(t)=X(t) H(t), \\
& X \text { is i.i.d., } \mathrm{E}[X]=0, \quad \mathrm{E}\left[X^{2}\right]=1, \\
& H(t)^{2}=\lambda S(t-1)^{2}+\tau H(t-1)^{2}, \\
& S \text { is i.i.d., } \quad \mathrm{E}[S]=0, \quad \mathrm{E}\left[S^{2}\right]=1, \quad \lambda, \tau \geq 0, \quad \tau<1,
\end{aligned}
$$

where i.i.d. stands for independent and identically distributed.

This model will be very close in spirit to Bollerslev's (1986) GARCH model if we set $S(t)=X(t)$. In this case, the difference between the GARCH processes is with respect to volatility function, where we use the past squared innovation $X(t-1)^{2}$ rather than the past squared realization $Y(t-1)^{2}$. This practice is also followed by, e.g., Nelson (1989) and Hsich (1989). Note that for covariance stationarity $\tau<1$ is sufficient for the process in (1), whereas $\lambda+\tau<1$ is required in case of $\operatorname{GARCH}(1,1)$.

The process defined in (1) exhibits the same properties as GARCH. More specifically, the process exhibits conditional heteroskedasticity, as past innovations and variances contribute to the current variance. This produces the clustering phenomenon (ii) as may be seen from

$$
H(t)^{2}-\sigma^{2}=\tau\left[H(t-1)^{2}-\sigma^{2}\right]+\lambda\left[S(t-1)^{2}-1\right],
$$

where $\sigma^{2}=\lambda /(1-\tau)$ is the unconditional variance of both $Y(t)$ and $H(t)$. The current variance overshoots the unconditional variance as a weighted average of past excesses in the innovation variances. For GARCH $(1,1)$, we 
have $^{3}$

$$
H(t)^{2}-\sigma^{2}=\tau\left[H(t-1)^{2}-\sigma^{2}\right]+\lambda\left[Y(t-1)^{2}-\sigma^{2}\right]
$$

where $\sigma^{2}=\omega /(1-\lambda-\tau)$. The only difference between (2) and (3) is the second term, which stems from using the innovations $S(t-1)$ or $X(t-1)$ rather than the realizations $Y(t-1)$ in the volatility function. The property (i) can be easily satisfied as well, by choosing $X$ to follow a fat-tailed distribution, like the Student- $t$ or stable distribution. In the latter case eqs. (2) and (3) make no sense, but evidently the clustering phenomenon is still present. With a finite variance $\sigma^{2}$, the unconditional distribution of $Y(t)$ cannot be stable and fat-tailed. Is it possible, though, that the above scheme (1) generates fat-tailed stable variates under slightly different conditions?

\section{Stable subordination with conditional scaling}

In order to answer the question at the end of the previous section, consider the following stochastic process called SSCS for ease of reference. ${ }^{4}$

Definition 1. The SSCS process is defined as the stationary solution of

$$
\begin{aligned}
& Y(t)=X(t) H(t)^{1 / \alpha}, \\
& H(t)=\lambda G(t-1)+\tau H(t-1), \quad \lambda>0, \quad 0 \leq \tau<1,
\end{aligned}
$$

where the $X(t)$ and $G(t-1)$ are each strictly stable i.i.d. random variables (r.v.) with characteristic exponents $\alpha, 0<\alpha \leq 2$, and $\beta, 0<\beta<1$, respectively, the $G(t)$ are nonnegative, and $X(t)$ and $G(t-j)$ are independent for all $j \geq 1$.

In comparison with eq. (1), the volatility function (5) - or scaling function in the context of stable d.f.'s - of the SSCS process still exhibits the clustering phenomenon. While past innovations do contribute to $H$, the relationship is slightly more complicated. It allows for more general patterns of clustering (see also footnote 6 below). Note that, while $X(t)$ and $G(t-j)$ are independent, Definition 1 does not rule out temporal dependency between $X(t)$ and $G(t)$. The following theorem ensures that the marginal distribution of $Y(t)$ is stable.

\footnotetext{
${ }^{3}$ The GARCH $(1,1)$ process is defined by $Y(t)=X(t) H(t), H(t)^{2}=\omega+\lambda Y(t-1)^{2}+$ $\tau H(t \quad 1)^{2}, \omega>0, \lambda \geq 0, \tau \geq 0, \lambda+\tau<1$, and $X(t) \sim \mathrm{N}(0,1)$.

${ }^{4}$ SSCS is the acronym for stable subordination with conditional scaling.
} 
Theorem 1. The unconditional distributions of the $Y(t)$ in eq. (4) are strictly stable with characteristic exponent $\alpha \beta$.

Proof. The proof proceeds in two steps. We first obtain the distribution of $H$ by convergence of an infinite convolution. In step 2 the unconditional distribution of $Y$ is derived as the product of two strictly stable variates.

Step 1. By repeated substitution the scaling function in eq. (5) can be rewritten as

$$
H(t)=\lambda \sum_{k=0}^{\infty} \tau^{k} G(t-k-1)+\lim _{n \rightarrow \infty} \tau^{n} H(t-n-1) .
$$

By Theorem 3 in Feller (1971, ch. VI.1) and recalling $0<\tau<1$, this is equivalent to

$$
\begin{aligned}
H(t) & \stackrel{\mathrm{d}}{=} \lambda\left[\sum_{k=0}^{\infty} \tau^{\beta k}\right]^{1 / \beta} G+\lim _{n \rightarrow \infty} \tau^{n} H(t-n-1) \\
& =\lambda\left[\frac{1}{1-\tau^{\beta}}\right]^{1 / \beta} G+\lim _{n \rightarrow \infty} \tau^{n} H(t-n-1),
\end{aligned}
$$

where $G$ is equal in distribution to $G(t)$.

The last term is zero in probability, and hence

$$
H(t) \stackrel{\mathrm{d}}{=} \lambda\left[\frac{1}{1-\tau^{\beta}}\right]^{1 / \beta} G
$$

By Theorem 2 in Feller (1971, ch. VI.1), $H(t)$ is positive, has a strictly stable distribution function with scale $\lambda\left[1 /\left(1-\tau^{\beta}\right)\right]^{1 / \beta}$ and characteristic exponent $\beta$.

Step 2. Given that $X(t)$ and $H(t)$ are strictly stable with characteristic exponents $\alpha$ and $\beta$, it follows from the product rule for stable variates [see, e.g., Feller (1971, ch. VI.2)] that

$$
Y(t)=X(t) H(t)^{1 / \alpha}
$$

is strictly stable with characteristic exponent $\alpha \beta$.

Note that the theorem holds for any unconditionally stable distributed $Y$, except the normal, by choosing $\alpha$ and $\beta$ appropriately. Therefore variates 
which are unconditionally leptokurtic stable may possess the clustering phenomenon (ii). The clustering derives from the volatility function that implies the conditional scale $H(t)^{1 / \alpha}$ which, when multiplied by the innovation $X(t)$, produces the subordinated process $Y(t)$ with stable marginals. Clark (1973) discusses stable subordinated stochastic processes in economics, but does not consider the possibility of conditional scaling.

Corollary 1. If the processes $\{G(t)\}$ and $\{X(t)\}$ are independent and $Y(t)$ follows the SSCS process of Definition 1, then $Y(t)$ satisfies the properties (i)-(iv).

Proof. Evidently, the fat-tail (i) and the clustering (ii) properties are satisfied. To obtain the additivity property (iii), note that conditional on the $I I(t)$ 's any sum $\sum_{j=0}^{n} Y(t-j)$ is strictly stable with scale

$$
\lambda^{1 / \alpha}\left\{\sum_{j=0}^{n-1}\left(\sum_{i=0}^{j} \tau^{j}\right) G(t-j-1)+\lambda^{-1}\left(\sum_{i=0}^{n} \tau^{i}\right) H(t-n)\right\}^{1 / \alpha}
$$

and characteristic exponent $\alpha$. By the additivity property of stable variates this scale is itself strictly stable distributed as well, with exponent $\beta$. Apply step 2 of the previous proof to conclude that $\sum_{j=0}^{n} Y(t-j)$ is strictly stable with characteristic exponent $\alpha \beta$. Therefore, $Y(t)$ and any finite sum of $Y(t)$ 's are of the same type. A similar argument shows this holds for any linear combination of $Y(t)$ 's. Property (iv) follows trivially as the stable distributions are in their own domain of attraction.

Remark 1. An easy proof of the third property in case the $X(t)$ 's are standard normal is given in Feller (1971, p. 176, fn. 7).

Remark 2. Because properiy (iii) holds, it also follows that all finite linear combinations of $Y(t)$ 's are strictly stable with the same characteristic exponent $\alpha \beta$. Theorem 2 of Dudley and Kanter (1974) then implies that $\{Y(t)\}$ is a stable stochastic process, and any subsequence of $Y(t)$ 's is multivariate stable. $^{5}$

Remark 3. Serial dependence in the mean can be introduced by adding a factor $\phi Y(t \quad 1), \phi^{2}<1$, to the right-hand side of eq. (4).

In view of the extant literature it is of interest to discuss the empirical implementation of the SSCS model. Consider the SSCS process of Definition

${ }^{5}$ Another implication of this is that the discrete-time SSCS stochastic process can be embedded in a continuous-time stochastic process [see Wolfe (1982)]. This is not possible for, e.g., the ARCH process. 
Table 1

Parameter estimates for the unconditional stable d.f. ${ }^{\text {a }}$

\begin{tabular}{llcc}
\hline Currencies & $\begin{array}{c}\text { Frequency } \\
\text { of data }\end{array}$ & $\begin{array}{c}\text { Scale } \\
\hat{s}\end{array}$ & $\begin{array}{c}\text { Characteristic } \\
\text { exponent } \widehat{\alpha \beta}\end{array}$ \\
\hline Canadian/U.S. dollar & Day & 0.00127 & 1.747 \\
German mark/U.S. dollar & Month & 0.00681 & 1.560 \\
Japanese yen/U.S. dollar & Day & 0.00339 & 1.624 \\
& Month & 0.02122 & 1.686 \\
& Day & 0.00234 & 1.266 \\
& Month & 0.01626 & 1.366 \\
\hline
\end{tabular}

${ }^{a}$ Estimates as reported in Boothe and Glassman (1987, p. 309).

1, suppose that $G(t)$ and $X(t)$ are independent, and let $X(t)$ have a standard normal d.f. such that $\alpha=2$ (in the spirit of Engle's original ARCH process). How can the parameters $\beta, \lambda$, and $\tau$ be estimated? The literature on speculative prices abounds with estimates of the unconditional d.f. of the returns. Table 1 contains some typical example estimates for the spot foreign exchange rate returns as reported in a recent survey by Boothe and Glassman (1987). From the last column of this table, an estimate for $\beta$ is easily calculated by division of $\widehat{\alpha \beta}$ with the maintained hypothesis $\alpha=2$. From eqs. (4) and (6) the unconditional scale is found as

$$
s=\lambda^{1 / \alpha}\left\{\sum_{j=0}^{n-1}\left(\sum_{i=0}^{j} \tau^{i}\right)^{\beta}+\left(\sum_{i=0}^{n} \tau^{i}\right)^{\beta} /\left(1-\tau^{\beta}\right)\right\}^{1 / \alpha \beta} .
$$

Hence, the scale estimates $\hat{s}$ reported in table 1 are a nonlinear combination of $\lambda, \tau, \alpha$, and $\beta$. On the basis of this information it is not possible to identify $\lambda$ and $\tau$ separately. Note, however, that the $\lambda$ can be divided out if one takes the ratio of two scale estimates based on two different frequencies. For months with $n+1$ days, from the following statistic $R_{n}$,

$$
R_{n}=\left[\frac{s(\text { month })}{s(\text { day })}\right]^{\alpha \beta}=\sum_{j=0}^{n-1}\left(\sum_{i=0}^{j} \tau^{i}\right)^{\beta}\left(1-\tau^{\beta}\right)+\left(\sum_{i=0}^{n} \tau^{i}\right)^{\beta},
$$

$\tau$ can be identified given an $\hat{\beta}$. Note that by induction on $n$, for all $n$, $\mathrm{d} R_{n} / \mathrm{d} \tau<0$ for $0<\tau<1$, and hence a simple grid-search procedure may be used to find $\hat{\tau}$. Once the conditional scaling coefficient $\hat{\tau}$ is known, the scale of the innovations $\hat{\lambda}$ is easily calculated from, say, the daily $\hat{s}$. Table 2 provides parameter estimates for the SSCS process on the basis of the 
Table 2

Parameter estimates for the SSCS process. ${ }^{\text {a }}$

\begin{tabular}{lccccc}
\hline Currencies & \multicolumn{2}{c}{ Imputed } & & & \\
\cline { 2 - 5 } Canadian/U.S. dollar & $\widehat{\alpha \beta}$ & $\hat{\beta}$ & $\hat{R}_{21}$ & $\hat{\tau}$ & $\hat{\imath}$ \\
German mark/U.S. dollar & 1.7 & 0.85 & 17.37 & 0.46 & 0.0008 \\
Japanese yen/U.S. dollar & 1.6 & 0.80 & 18.81 & 0.15 & 0.0029 \\
\hline
\end{tabular}

${ }^{a}$ Due to different $\alpha \beta$ estimates for different frequencies, some overall characteristic exponent has to be used in the calculations of $\tau$ and $\lambda$; this information is recorded in the first column. Grid search was used to calculate $\tau$ from the nonlinear eq. (8). Calculations are based on the presumption that a month contains $n+1=22$ trading days.

information contained in table 1 . The $\hat{\tau}$ values indicate the contribution of past scales to the current scaling coefficient. An indication for the persistence in the scaling and thus the importance of volatility clusters are the mean lag $\tau /(1-\tau)$, with values of $0.85,0.17$, and 0.56 , respectively, and the median lag which is zero in all cases [see Bollerslev (1986, pp. 311-312)]. This suggests that while past volatility does contribute to the current volatility, the effect evaporates fairly rapidly.

How do these estimates, the $\tau$ values in particular, compare to the existing evidence? We address two issues, the size of $\tau$ and the effects of temporal aggregation. To start with the latter issue, as Diebold (1988) shows on the basis of a central limit theorem argument, temporal aggregates of ARCH processes tend to normality. Empirically, Baillie and Bollerslev (1989) find that while GARCH effects are present in daily and weekly foreign exchange return data, these effects disappear in biweekly and monthly returns. A nice property of the SSCS process is that the effects of time aggregation can be explicitly calculated; i.e., no limit arguments are needed even though they do apply as stable distributions are in their own domain of attraction. Define $Z(-m)=Y(t-(m-1) k)+\cdots+Y(t-m k+1)$ for some periodicity $k \geq 2$ and $m=1,2, \ldots$, and study the behavior of the time-aggregated series $Z(-m)$. Note that $H(t)+\cdots+H(t-(k-1))$ is equal in distribution to $R_{k-1}^{1 / \beta} H(t)$, where $R_{k}$ was defined in (8). The time-aggregated SSCS process can then be written as

$$
\begin{aligned}
& Z(-m) \stackrel{\mathrm{d}}{=} X(-m) R_{k-1}^{1 / \alpha \beta} H(-m)^{1 / \alpha}, \\
& H(-m) \stackrel{\mathrm{d}}{=} \lambda c G(-m-1)+\tau^{k} H(-m-1),
\end{aligned}
$$

where $X, G$, and $H$ are distributed as before and $c$ is a positive nonlinear function of $\tau$. The important thing to note is the factor $\tau^{k}$ in the scaling 
function. It is not hard to show that $\lim \tau^{k} R_{k-1}^{1 / \beta}=0$ as $k \rightarrow \infty$. Thus the clustering effect is reduced due to temporal aggregation, as in the case of GARCH. Empirically, for the estimates in table 2, the clustering effect is virtually zero on a fortnightly scale.

In comparison with, e.g., the GARCH estimates reported in Bollerslev (1987), Baillie and Bollerslev (1989), and Hsieh (1989), our $\hat{\tau}$ values point to a lower persistence [typically $\lambda+\tau$ as in eq. (3) are close to one in these references]. Interestingly Hsieh also estimates the exponential GARCH model and finds that the autoregressive parameter in the volatility function is significantly below one. This may be due to the logarithmic specification which reduces the effect of outliers in the volatility function. Thus, an explanation for the relatively low $\tau$ values we find may be that the stable model is 'robust' against outliers. This point may be of interest for future research, but is outside the scope of the present paper.

Up to this point the innovations $G$ in the volatility function (5) have been considered to be independent from the past innovations $X$ or realizations $Y$. It is of interest to relax this assumption. First consider dependence of $G(t)$ on $X(t)$. Specifically, suppose that the dependence takes the following form:

$$
G(t)=F_{2}^{-1}\left(F_{1}(X(t))\right)
$$

where $F_{1}$ is the d.f. of $X, F_{2}$ is the d.f. of $G$, and $X$ and $G$ are both strictly stable r.v.'s as stated in Definition 1. Therefore, $F_{1}$ and $F_{2}$ are continuous [see, e.g., Feller (1971, ch. VI.13)], have the same range, and are monotone. It follows that the composite function in eq. (9) defines a strictly stable r.v. with characteristic exponent $\beta$. It is easy to see that, as $X(t)$ and $G(t-j)$ are independent r.v.'s, Theorem 1 is applicable and hence the unconditional distribution of $Y(t)$ is strictly stable with characteristic exponent $\alpha \beta$. As $\alpha \beta<2$, the fat-tail property (i) holds again. From the discussion in the previous section and the Definition 1 the stochastic process also exhibits the cluster property (ii).

Corollary 2. The SSCS process of Definition 1, amended with the dependency structure as in eq. (9), exhibits properties (i) and (ii).

To foster the reader's intuition, the following process provides a tractable example:

$$
\begin{aligned}
& Y(t)=X(t) H(t)^{1 / 2}, \quad X(t) \text { is i.i.d. standard normal, } \\
& H(t)=\frac{1}{X(t-1)^{2}}+\tau^{2} H(t-1), \quad 0 \leq \tau<1
\end{aligned}
$$


As $X$ follows the standard normal distribution with density $f_{\alpha}(x)$, i.e., it is a strictly stable variate with characteristic exponent $\alpha=2$, the composite function $1 / X^{2}$ is a strictly stable variate with characteristic exponent $\beta=\frac{1}{2}$ and has density

$$
f_{\beta}(g)=(2 \pi)^{-1 / 2} g^{-3 / 2} \mathrm{e}^{-1 / 2 g},
$$

see Feller (1971, ch. II.4). From step 1 in the proof of Theorem 1, $H$ has density [note the $\tau^{2}$ in (10)]

$$
f_{\beta}(h)=(2 \pi)^{-1 / 2}(1-\tau)^{-1} h^{-3 / 2} \exp \left[2(1-\tau)^{2} h\right]^{-1} .
$$

The unconditional density of $Y$ can now be found as a mixture of the normal

$$
f_{\alpha \beta}(y)=\int f_{\alpha}(y \mid h) f_{\beta}(h) \mathrm{d} h=1 / \pi\left[(1-\tau)^{-1}+(1-\tau) y^{2}\right] .
$$

The unconditional distribution of $Y$ is Cauchy, i.e., is stable with characteristic exponent $\alpha \beta=1{ }^{6}$

This example is also instructive in showing that this variant of the SSCS process does not exhibit the additivity property (iii), cf. Corollary 1 and 2 . Suppose that $\tau=0$ in (10) and hence the process can simply be written as $Y(t)=X(t) / X(t-1)$. It is immediate that the unconditional distribution of $Y(t)$ is Cauchy, given that the $X(t)$ 's are i.i.d. standard normal. Straightforward calculations show that the joint density of two adjacent $Y$ 's, say $A=Y(t)$ and $B=Y(t-1)$, reads

$$
f(a, b)=(2 \pi)^{-1} a^{-2}\left(1+b^{2}+a^{-2}\right)^{-3 / 2} .
$$

For $\{Y(t)\}$ to be a stable process, a necessary condition is that each univariate marginal - i.e., including all linear combinations - is stable with the same index; see, e.g., Dudley and Kanter (1974). It is relatively straightforeward to show that the marginals $f(a)=\int f(a, b) \mathrm{d} b$ and $f(b)=\int f(a, b) \mathrm{d} a$ are Cauchy, i.e., have index one. How about the sum? Let $Q=A+B$, and evaluate the density of the sum $f(a)=\int f(a-b, b) \mathrm{d} b$. This integral is hard to integrate due to the fractional power of the denominator. However, it is easily shown that $f(q)$ is symmetric around $q=0$. Moreover, some tedious

\footnotetext{
${ }^{6}$ Interestingly, as was pointed out by a referee, the process (10) constitutes an example of 'negative clustering' in analogy with the effect of negative serial correlation. If the previous innovation was high in absolute terms, it lowers the current conditional variance and vice versa. Hence, the volatility tends to alternate.
} 
calculus shows

$$
\begin{aligned}
f^{\prime \prime}(0) & =-\frac{3}{\pi} \int_{0}^{\infty}\left\{\frac{a^{3}}{\left[1+a^{2}+a^{4}\right]^{5 / 2}}-5 \frac{a^{7}}{\left[1+a^{2}+a^{4}\right]^{7 / 2}}\right\} \mathrm{d} a \\
& =2 / 27 \pi>0 .
\end{aligned}
$$

But the Cauchy distribution that is symmetric around zero has $f^{\prime \prime}(0)<0$. Hence, $f(q)$ is not Cauchy. In fact, by numerical integration $f(q)$ was found to be bimodal. It follows that property (iii) cannot be satisfied.

As a last example of processes with unconditional stable variates which exhibit volatility clustering, consider the following bilinear model: ${ }^{7}$

$$
\begin{aligned}
& Y(t)=X(t) H(t)^{1 / 2}, \\
& H(t)^{2}=\lambda Y(t-1)^{2} Q(t)^{2 / \beta},
\end{aligned}
$$

where the $X(t)$ are i.i.d. standard normal distributed and the $Q(t)$ are i.i.d. strictly stable distributed with characteristic exponent $\frac{1}{2}$.

Note that the volatility function now directly depends on the past realization like in Engle's (1982) original ARCH process. The process also has the format of a random coefficient model [cf. Wolff (1988)]. It can be checked by using the product rule for stable r.v.'s that the unconditional distribution of $Y(t)$ is stable with characteristic exponent $\beta$.

\section{Summary}

The paper shows that studies which concentrated on the unconditional distribution of asset returns and hypothesized a stable d.f., are not necessarily inconsistent with ARCH-type processes that exhibit clusters of volatility. This has some importance for modeling the distribution of returns on financial assets. Typically the returns are leptokurtic and exhibit clusters of volatility. The SSCS model offers a means to nest the finite-variance and infinite-variance stable alternative within an ARCH-type scheme. It was noted that while the unconditional distribution of the SSCS variates can be stable, sums of these variates may or may not be identical in distribution to the summands. Thus the SSCS process may fail the additivity property. In the latter case, dependence of the volatility function on previous innovations and realizations was considered. Example processes of both cases were given, as well as some hints towards empirical implementation.

${ }^{7}$ On bilinear models, see Granger and Andersen (1978). 


\section{References}

Akgiray, V. and G.G. Booth, 1988, The stable-law model of stock returns, Journal of Business and Economic Statistics, 51-57.

Baillie, R.T. and T. Bollerslev, 1989, The message in daily exchange rates: A conditional variance tale, Journal of Business and Economic Statistics, 297-305.

Blattberg, R.C. and N.J. Gonnedes, 1974, A comparison of the stable and student distributions as statistical models for stock prices, Journal of Business, 244-280.

Bollerslev, T., 1986, Generalized autoregressive conditional heteroskedasticity, Journal of Econometrics, 307-327.

Bollerslev, T., 1987, A conditionally heteroskedastic time serics model for spcculative priccs and rates of return, Review of Economics and Statistics, 542-547.

Bollerslev, T. and R.F. Engle, 1986, Modelling the persistence of conditional variances, Econometric Reviews, 1-50.

Boothe, P. and D. Glassman, 1987, The statistical distribution of exchange rates, Journal of International Economics, 297-319.

Clark, P.K., 1973, A subordinated process model with finite variance for speculative prices, Econometrica, 135-155.

Diebold, F.X., 1987, Temporal aggregation of ARCH processes and the distribution of asset returns, Special studies paper 200 (Federal Reserve Board, Washington, DC).

Diebold, F.X., 1988, Empirical modeling of exchange rate dynamics (Springer Verlag, Berlin).

Dudley, R.M. and M. Kanter, 1974, Zero-one laws for stable measures, Proceedings of the American Mathematical Society, 245-252.

Engle, R.F., 1982, Autoregressive conditional heteroscedasticity with estimates of the variance of the U.K. inflation, Econometrica, 987-1008.

Feller, W., 1971, An introduction to probability theory and its applications, Vol. II (J. Wilcy, New York, NY).

Granger, C.W.J. and A.P. Andersen, 1978, An introduction to bilinear time series (Vandenhoeck \& Ruprecht, Göttingen).

Haan, L. de, S.I. Resnick, H. Rootzen, and C.G. de Vries, 1989, Extremal behaviour of solutions to a stochastic difference equation with applications to ARCH processes, Stochastic Processes and their Applications, 213-224.

Hsieh, D.A., 1989, Modeling heteroscedasticity in daily foreign exchange rates, Journal of Business and Economic Statistics, 307-317.

Kon, S., 1984, Models of stock returns: A comparison, Journal of Finance, 147-165.

Mandelbrot, B., 1963, The variation of certain speculative prices, Journal of Business, 394-419.

McCulloch, J.H., 1985, Interest-risk sensitive deposit insurance premia, stable ACH estimates, Journal of Banking and Finance, 137-156.

Nelson, D.B., 1989, Conditional heteroskedasticity in asset returns: A new approach, Mimeo. (University of Chicago, Chicago, IL).

Tucker, A.L. and L. Pond, 1988, The probability distribution of foreign exchange price changes: Tests of candidate processes, Review of Economics and Statistics, 638-647.

Westerfield, J.M., 1977, An examination of foreign exchange risk under fixed and floating rate regimes, Journal of International Economics, 181-200.

Wolfe, S.J., 1982, On a continuous analogue of the stochastic difference equation $X_{n}=\rho X_{n-1}+$ $B_{n}$, Stochastic Processes and their Applications, 301-312.

Wolff, C.C.P., 1988, Autoregressive conditional heteroscedasticity: A comparison of ARCH and random coefficient models, Economics Letters, 141-143. 